\title{
SOME PROBLEMS ON 3-DIMENSIONAL MANIFOLDS
}

\section{D. PAPAKYRIAKOPOULOS}

\section{Generalities}

1. Introduction. One of the well-known problems in Topology is the classification problem of closed $n$-dimensional manifolds.

An $n$-manifold ( $n$-dimensional manifold) is a connected separable metric space each of whose points has a closed neighborhood homeomorphic to a closed $n$-cell. So we consider both manifolds with boundary and manifolds without boundary. A closed n-manifold is a compact $n$-manifold without boundary.

Classification means to define an infinite sequence of closed $n$ manifolds $M_{1}, M_{2}, M_{3}, \cdots,{ }^{1}$ such that any two of these are not homeomorphic, but any closed $n$-manifold $M$ is homeomorphic with one of them. We emphasize that, we do not ask to find a method to decide with which of the model manifolds is $M$ homeomorphic. We only want to know whether $M$ is included in this sequence. Of course we do not ask to find an effective procedure, because such may not exist.

The classification problem was solved long ago for $n=2$, i.e. for closed surfaces, ${ }^{2}[22, \S \S 37-39$, pp. $130-142]$. So, as usual in Mathematics, one tries to solve the problem for the next dimension $n=3$, in the hope that he will find a general method working for any dimension. This is the reason we restrict ourselves from now on to the case $n=3$.

The classification problem has been solved not only for closed surfaces, but also for compact nonclosed ones [22, §40, pp. 142-144; 10, pp. 151-158]. See also [10, p. 171, 11. 12-16].

We concentrate our attention on the classification problem of closed 3-manifolds, and for the time being we do not consider the classification problem for nonclosed 3-manifolds, because this last problem seems to be much more complicated, see No. 21.

2. Generalities. As is well known, the classification problem is solved for $n=2$ by cutting the surface along simple ${ }^{3}$ curves. So the question arises naturally: Can we solve the classification problem for

An address delivered before the Annual Meeting of the Society in Cincinnati, Ohio, on January 30,1958, by invitation of the Committee to Select Hour Speakers for Annual and Summer Meetings; received by the editors March 18, 1958.

${ }_{1}^{1}$ To define $M_{i}$ means to give a model of $M_{i}$, i.e. a way of constructing $M_{i}$.

2 Numbers in brackets refer to the bibliography at the end of the paper.

8 I.e. without self-intersections. 
$n=3$, by cutting the closed 3-manifold along surfaces without self-intersections? A close examination of this problem suggests that we have to face the following two problems.

(2.1) To define a family of surfaces, possibly with self-intersections, having certain properties.

(2.2) To select from the family a surface that has no self-intersections, i.e. we need to prove the existence in the family of a surface without self-intersections.

These are generalities, and they will be clarified in II.

\section{The SPHere theorem, Dehn's lemma, AND the LOOP THEOREM}

3. The sphere theorem. From now on everything will be considered from the semi-linear point of view, i.e. any 3-manifold will be considered with a fixed triangulation, which is permissible according to E. E. Moise's $[13 ; 14]$ and R. H. Bing's $[1 ; 2]$ work, any curve will be considered as polygonal, any surface as polyhedral, and so on.

Let $M$ be a 3-manifold, such that $\pi_{2}(M) \neq 0$. This means that there exist in $M 2$-spheres, with self-intersections (singularities), which are noncontractible in $M$. Thus a family, required by (2.1), is well defined. Now in connection with (2.2) the question is: does the family contain a surface without self-intersections? This poses a certain problem, which may be called the sphere problem.

To the best of my knowledge, the first to attempt a problem of this kind was H. Kneser in 1928, [11, p. 257]. He remarked that if $S$ is a 2-sphere (without singularities) in $M$, such that $M-S$ consists of two components $M^{\prime}$ and $M^{\prime \prime}$, then

$$
\pi_{1}(M) \approx A * B,
$$

where $\pi_{1}\left(M^{\prime}\right) \approx A$ and $\pi_{1}\left(M^{\prime \prime}\right) \approx B$. Then he tried to prove the following theorem, which is the converse of his remark.

(3.2) If (3.1) holds, then there exists a 2-sphere without singularities $S$ in $M$, such that $M-S$ consists of two components $M^{\prime}$ and $M^{\prime \prime}$, where $\pi_{1}\left(M^{\prime}\right) \approx A$ and $\pi_{1}\left(M^{\prime \prime}\right) \approx B$.

However his proof does not seem to be conclusive. See Nos. 12, 15, and especially Nos. 17 and 20.

In 1937 appeared the paper of S. Eilenberg [5], which initiated the asphericity problem of knots, see No. 12. This paper inspired the 1939 paper of J. H. C. Whitehead [28], on p. 161 of which the following problem or conjecture is contained.

(3.3) A nonempty proper open connected subset $U$ of the 3-sphere is

\footnotetext{
$4 *$ means free product.
} 
aspherical, if and only if any 2-sphere in $U$ bounds a 3-cell belonging to $U$.

It was precisely this conjecture which stimulated the present author to prove during the summer of 1956 the following sphere theorem $[18$, p. 1].

(3.4) Let $M$ be an orientable 3-manifold, compact or not, with boundary which may be empty, such that $\pi_{2}(M) \neq 0$, and which can be semilinearly imbedded in a 3-manifold $N$, having the following property: the commutator quotient group of any nontrivial (but not necessarily proper) finitely generated subgroup of $\pi_{1}(N)$ has an element of infinite order (n.b. in particular this holds if $\left.\pi_{1}(N)=1\right)$. Then there exists a 2-sphere $S$ semi-linearly imbedded in $M$, such that ${ }^{5} S \not 0$ in $M$.

In October $1957 \mathrm{~J}$. W. Milnor proved a more general sphere theorem. Finally in December 1957 J. H. C. Whitehead [29] proved the sphere theorem in complete generality.

SPHeRE THEOREM. Let $M$ be an orientable 3-manifold, compact or not, with boundary which may be empty, such that $\pi_{2}(M) \neq 0$. Then there exists a 2-sphere $S$ semi-linearly imbedded in $M$, such that $S \not 20$ in $M$.

Both Milnor's and Whitehead's proofs are modifications of the present author's proof $[18, \S 5, \mathrm{pp} .15-18]$. The sphere theorem does not hold generally for nonorientable 3 -manifolds as the example ${ }^{6}$ $P^{2} \times S^{1}$ shows.

4. Dehn's lemma. Parallel to the sphere theorem lies another proposition known as Dehn's lemma.

Dehn's Lemma. Let $M$ be a 3-manifold, compact or not, with boundary which may be empty, and in $M$ let $D$ be a 2-cell with self-intersections (singularities), having as boundary the simple ${ }^{8}$ closed polygonal curve $C$, and such that there exists a closed neighborhood of $C$ in $D$ which is an annulus (i.e. no point of $C$ is singular). Then there exists a 2-cell $D_{0}$ with boundary $C$, semi-linearly imbedded in $M$.

Dehn's lemma was included in a 1910 paper of M. Dehn [4, p. 147], but in 1928 H. Kneser [11, p. 260], observed that Dehn's proof contained a serious gap. In 1935 and 1938 appeared two papers by I. Johansson [8; 9], on Dehn's lemma. In the second one, p. 659, he proved that, if Dehn's lemma holds for all orientable 3-manifolds, it then holds for all nonorientable ones. During the summer of 1956 the present author proved that Dehn's lemma holds for all orientable 3manifolds [18, §4, pp. 13-15].

${ }^{5} \simeq$ means homotopic to.

${ }^{6} P^{2}$ is the real projective plane, and $S^{1}$ is the 1 -sphere. 
In a forthcoming paper of Arnold S. Shapiro and J. H. C. Whitehead [30] a simplified proof of Dehn's lemma is given. There, is proved also an extension of the lemma, for Dehn discs with more than one boundary curve, see $[18$, p. 24 , Problem 2$]$.

5. The loop theorem. Another proposition related to the sphere theorem and Dehn's lemma is the following

Loop Theorem. Let $M$ be a 3-manifold which may or may not be compact, with boundary $N$ formed by a number $(>0, \leqq \infty)$ of surfaces closed or not. Let $L$ be a loop belonging to an open set $U$ of an orientable component $N^{\prime}$ of $N$, such that $L \simeq 0$ in $M$ and $\chi_{0}$ on $N$. Then there exists a simple loop $L_{0}$ in $U$, such that $L_{0} \simeq 0$ in $M$ and $\not 20$ on $N$.

This theorem was contained implicitly in the 1928 paper of $\mathrm{H}$. Kneser mentioned twice above. Actually, the "Hilfssatz" [11, p. 248] has to be split into the loop theorem and Dehn's lemma. We would like to emphasize that the loop theorem is independent of Dehn's lemma, i.e. it does not follow from Dehn's lemma, and moreover its proof has its own difficulties. It seems that J. H. C. Whitehead was in 1937 the first to observe this splitting. Actually, in $[27$, p. 65], the following lemma is proved, which is a special case of the loop theorem.

(5.1) Let us suppose that $M, N, N^{\prime}$ are as in the loop theorem, and let $L$ be a simple loop on $N^{\prime}$ such that $L^{8} \simeq 0$ in $M$, where $s$ is a natural number. Then $L \simeq 0$ in $M$.

Finally this author proved the loop theorem in complete generality [16, pp. 285-293]. However, I reproved (5.1) and I had to make use of it in my proof. This is Lemma (9.3), p. 287.

6. Relation between the sphere theorem, Dehn's lemma, and the loop theorem. Looking more closely at these three propositions we recognize that they are of the same kind, namely: given a certain geometric entity with certain properties, to find ${ }^{7}$ a simplest possible geometric entity with the same properties. This needs some explanation.

In the sphere theorem the geometric entity is a 2 -sphere with singularities which lies in $M$, and its property is to be noncontractible in $M$. From this we construct a 2 -sphere (without singularities) which lies in $M$ and is noncontractible in $M$, i.e. we find a simplest possible geometric entity with the same properties.

In Dehn's lemma the geometric entity is the 2-cell with singularities which lies in $M$, and its properties are that it has boundary $C$, and its singularities are far away from the boundary. From these we construct a 2-cell (without singularities) which lies in $M$ and has bound-

${ }^{7}$ To find means here to construct or prove the existence of. 
ary $C$, i.e. we find a simplest possible geometric entity with the same properties.

In the loop theorem the geometric entity is the loop $L$ which lies in $U$, and its properties are that it is $\simeq 0$ in $M$ and $\not 0$ on $N$. From this we construct a simple ${ }^{3}$ loop which lies in $U$, is $\simeq 0$ in $M$ and $\neq 0$ on $N$, i.e. we find a simplest possible geometric entity with the same properties.

So actually the sphere theorem, Dehn's lemma, and the loop theorem are of the same kind. However, their relation is deeper, and this will be clarified in III, where we will give brief sketches of their proofs.

\section{SKETCH OF PROOFS}

7. Proof of the loop theorem. Let us consider the following diagram

$$
\begin{array}{ccc}
\tilde{M} & \supset \tilde{N}^{\prime} \supset \tilde{L} \\
p \downarrow & \downarrow & \downarrow \\
M & \supset N^{\prime} \supset L
\end{array}
$$

where $p: \tilde{M} \rightarrow M$ is the universal covering of $M, \tilde{N}^{\prime}$ is a component of bd $\tilde{M}$ lying over $N^{\prime}$, and $\tilde{L}$ is a loop on $\tilde{N}^{\prime}$ covering $L$ just once. This is possible, because $L \simeq 0$ in $M$. It is no loss of generality to suppose that, $L$ has only "double" points, where two branches of $L$ are crossing each other. Let $d(L)$ and $d(\tilde{L})$ be the number of double points of $L$ and $\tilde{L}$ respectively. Then

$$
d(L)=d(\tilde{L})+\frac{1}{2} \sum d(\tilde{L}, \tau \tilde{L})
$$

where the sum ranges over all covering translations $\tau$ of $p: \tilde{M} \rightarrow M$, which are different from the identity, and $d(\tilde{L}, \tau \tilde{L})$ is the number of common points of $\tilde{L}$ and $\tau \tilde{L}$. We easily obtain from $L$ a new loop $L^{\prime}$ lying in $U, \simeq 0$ in $M$, and $\not 0$ on $N$, such that

$$
d\left(\tilde{L}^{\prime}\right)=0
$$

where $\tilde{L}^{\prime}$ is a loop on $\tilde{N}^{\prime}$ covering $L^{\prime}$ just once.

From now on begin the main difficulties of the problem. We have to use the fact that $\tilde{N}^{\prime}$ can be topologically imbedded in a 2-sphere, and then we have to make a detailed study comparing the position of $\tilde{L}^{\prime}$ with that of $\tau \tilde{L}^{\prime}$. This is a rather delicate argument, and therefore we refer the reader to [16, pp. 285-293].

We would like to observe, that J. H. C. Whitehead did not use

\footnotetext{
$8 \mathrm{bd}=$ boundary
} 
covering spaces in his proof of (5.1), and perhaps therefore he obtained only a special case of the loop theorem.

8. Proof of Dehn's lemma. Having observed in No. 6 that the loop theorem and Dehn's lemma are problems of the same kind, and having proved the loop theorem, the question arises naturally: can we use the same method, or at least a modification of it, to prove Dehn's lemma? The answer is affirmative and we are now going to explain this method.

Performing certain rather elementary geometric operations, we may suppose that the only singularities of the Dehn $d i s c^{9} \mathrm{D}$ are double curves, along which two sheets cross, and triple points at which three sheets cut $[18$, Nos. 2-3, pp. 3-6]. Let $d(D)$ and $t(D)$ be the number of double curves and triple points of $D$, respectively. The ordered pair $(t(D), d(D))$ will serve as complexity of $D$.

Let us consider the following diagram

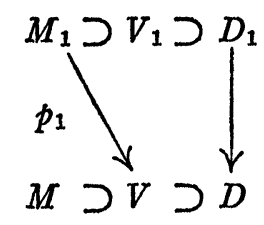

where $D$ is the Dehn $\operatorname{disc}^{9}$ with boundary $C, V$ is a prismatic neighborhood of $D$ in $M$, and $p_{1}: M_{1} \rightarrow V$ is the universal covering of $V$. Let $D_{1}$ be a Dehn disc in $M_{1}$ covering $D$ just once. Then, see [17, formula on p. 171],

$$
d(D)=d\left(D_{1}\right)+\frac{1}{2} \sum d\left(D_{1}, \tau D_{1}\right)
$$

where the sum ranges over all covering translations $\tau$ of $p_{1}: M_{1} \rightarrow V$, which are different from the identity, and $d\left(D_{1}, \tau D_{1}\right)$ is the number of common closed curves of $D_{1}$ and $\tau D_{1}$. Thus the following holds.

(8.3) $d\left(D_{1}\right) \leqq d(D)$, and the equality holds if and only if $V$ is simply connected.

Let $V_{1}$ be a prismatic neighborhood of $D_{1}$ in $M_{1}$. So we have the diagram (8.1). Comparing (7.1) and (7.2) with (8.1) and (8.2), we see the differences and the similarities.

We can repeat again and again the above construction, and so we obtain the following diagram

\footnotetext{
${ }^{9} \mathrm{~A}$ Dehn disc means a 2-cell whose singularities, if any, are far away from the boundary.
} 

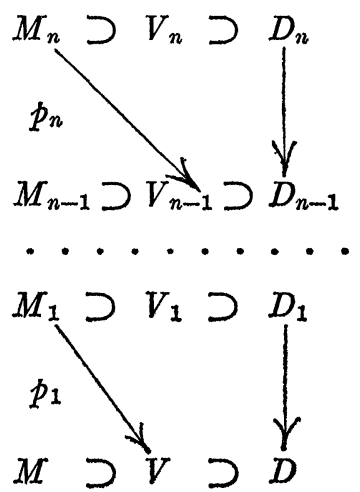

called a tower over $D \subset M$, and the sequence

$$
d(D) \geqq d\left(D_{1}\right) \geqq \cdots \geqq d\left(D_{n}\right) \geqq \cdots \cdots 0,
$$

by (8.3). Thus by (8.3), there is a number ${ }^{10} n \geqq 0$, such that $d\left(D_{i}\right)$ $>d\left(D_{i+1}\right)$ for $i<n$, and $d\left(D_{j}\right)=d\left(D_{j+1}\right)$ for $j \geqq n$; i.e. the tower is trivial after the crucial value $n$, which is called the height of the tower.

We now have to consider the following two cases $d\left(D_{n}\right)>0$ and $d\left(D_{n}\right)=0$. In both cases performing certain operations at the $n$th level, and projecting by the map $p_{1} \cdots p_{n}$ we obtain a new Dehn $\operatorname{disc}^{9} D^{\prime}$ in $M$, with boundary $C$, which is simpler than $D$. This means that either $t\left(D^{\prime}\right)<t(D)$, or if $t\left(D^{\prime}\right)=t(D)$ then $d\left(D^{\prime}\right)<d(D)$.

We would like to emphasize, that the case $d\left(D_{n}\right)>0$ is rather geometric $\left[18\right.$, No. 16, pp. 14-15], and the case $d\left(D_{n}\right)=0$ is rather algebraic $[18$, No. 17, p. 15$],{ }^{11}$ and actually here appears the main difficulty of Dehn's lemma. Compare this with the remarks after (7.3).

In the same way we obtain from $D^{\prime}$ a new Dehn disc $D^{\prime \prime}$ in $M$, with boundary $C$, which is simpler than $D^{\prime}$, and so on. Finally, after a finite number of repetitions of this construction, we obtain a Dehn disc $D^{(m)}$ in $M$, with boundary $C$ and complexity $(0,0)$. This means that $D^{(m)}$ has neither triple points nor double lines. Hence $D^{(m)}$ is the required 2-cell $D_{0}$, without singularities.

Arnold S. Shapiro suggested considering 2-sheeted coverings, instead of universal ones, in the construction of the tower $[18$, p. 24 , Problem 5].

In a forthcoming paper of Arnold S. Shapiro and J. H. C. Whitehead [30] a simplified proof of Dehn's lemma is given using 2-sheeted

${ }^{10} M_{0}=M, V_{0}=V, D_{0}=D$.

1 That No. 17 is based on Nos. 11-12, pp. 11-13. 
coverings. There, is proved also an extension of the lemma, for Dehn discs with more than one boundary curve, see [18, p. 24, Problem 2].

9. Proof of the sphere theorem. Let us consider first my proof of (3.4), [18, §5, pp. 15-18]. The method is similar to that of Dehn's lemma explained in No. 8. Namely, performing certain rather elementary geometric constructions, we may suppose that the only singularities of a 2-sphere with self-intersections $D$ are double curves and triple points, whose number is $d(D)$ and $t(D)$ respectively. We consider again the diagram (8.1), where now $D$ means a 2 -sphere with singularities, such that $D \Varangle 0$ in $M$. Then formula (8.2) and proposition (8.3) hold. We construct the tower (8.4), consider the height $n \geqq 0$ of it, and we have again the two cases $d\left(D_{n}\right)>$ or $=0$. Performing certain operations at the $n$th level and projecting by the map $p_{1} \cdots p_{n}$ we obtain a new 2-sphere with singularities $D^{\prime}$ in $M$, such that $D^{\prime} \not 0$ in $M$, and which is simpler than $D$, as in the proof of Dehn's lemma. However, here we have to be especially careful to secure the condition $D^{\prime} \not 0$ in $M$. This can be done using Poincaré duality and standard Hurewicz theorems [18, p. 17, Lemma (22.5)]. In the same way we obtain from $D^{\prime}$ a new 2-sphere with singularities $D^{\prime \prime}$ in $M$, such that $D^{\prime \prime} \not \chi 0$ in $M$, which is simpler than $D^{\prime}$ and so on. Finally, after a finite number of repetitions of this construction, we obtain a 2-sphere with singularities $D^{(m)}$ in $M$, such that $D^{(m)} \not 0$ in $M$, and which has complexity $(0,0)$. This means that $D^{(m)}$ has neither triple points nor double lines. Hence $D^{(m)}$ is the required 2-sphere $S$, without singularities.

Theorem (3.4) contains a rather restrictive condition. Namely, the condition of the imbeddability of $M$ in $N$, which has a certain property. This condition is used only in the special case where the height of the tower $(8.4)$ is $n=1$, and $d\left(D_{1}\right)=0,[18$, p. $18,11.1-14]$.

In December 1957 J. H. C. Whitehead freed the theorem from this condition, and so we now have the sphere theorem in complete generality. He constructs a tower (8.4) in the following way. Let us consider the diagram (8.1) as defined above. Let $\tau$ be a covering translation of $p_{1}: M_{1} \rightarrow V$ different from the identity, and such that $\tau D_{1}$ meets $D_{1}$. Then in (8.4), the covering $p_{1}: M_{1} \rightarrow V$ is not the universal one as in our construction, but a covering corresponding to the subgroup of $\pi_{1}(V)$ generated by $\tau$. All coverings $p_{i}: M_{i} \rightarrow V_{i-1}, i=2, \cdots$, $n$, are universal as in our construction. Finally he stops the tower at a height $n \geqq 0$, such that $\pi_{1}\left(V_{n}\right)$ is finite, while we essentially stopped the tower at a height $n \geqq 0$, such that $\pi_{1}\left(V_{n}\right)=1$. These are Whitehead's modifications, and after that everything works smoothly following our method. 
It was suggested by R. H. Fox to use also other coverings, instead of universal ones [18, p. 24, Problem 5]. However Whitehead showed the importance of the nonuniversal coverings.

\section{Applications}

10. Applications of the loop theorem. As it is well-known any closed orientable 3-manifold can be obtained from two solid tori of the same genus $(\geqq 0)$ by matching their boundaries $[22$, p. 219 , Satz $]$.

Solid torus of genus ${ }^{12} h(\geqq 0)$ means a 3 -cell with $h$ solid handles. This is a compact orientable 3-manifold $M$, whose boundary $N$ is an orientable surface of genus $h$, such that $\pi_{1}(M, N)=1$. The importance of the solid tori suggests the desirability of obtaining a topological characterization of them; and, according to the above remark, the following conjecture arises naturally.

(10.1) Let $M$ be a compact 3-manifold, whose boundary $N$ is an orientable surface of genus $h(\geqq 0)$, such that $\pi_{1}(M, N)=1$. Then $M$ is a solid torus of genus $h$.

We observe that an immediate consequence, of the special case $h=0$, of $(10.1)$ is the

Cell Conjecture. A simply connected compact 3-manifold, whose boundary is a 2-sphere, is a 3-cell.

This is equivalent to the well-known

Poincare Conjecture. A simply connected closed 3-manifold is a 3-sphere.

Some years ago I was working on Poincaré conjecture, and I tried to prove it by proving (10.1). But I failed, and I may say that I am now convinced that this is not the way to attack Poincaré conjecture. However, the loop theorem, Dehn's lemma, Poincaré conjecture, and some results from algebraic topology imply $(10.1)$, see $[16$, p. 297 , Theorem (19.1)]. This was the reason I worked on the loop theorem, whose proof led me to the proof of Dehn's lemma and the sphere theorem.

Another application of the loop theorem can be made in proving H. Kneser's "Hilfssatz" [11, p. 248]. For other applications of the loop theorem see [19, §5, pp. 91-92], and [16, pp. 298-299].

11. Applications of Dehn's lemma. Dehn's lemma was introduced by M. Dehn in 1910 to prove an algebraic characterization of the unknotted knots in the 3-sphere $S^{3}$.

By a knot $K$ in $S^{3}$ we mean a simple ${ }^{8}$ closed polygonal curve in $S^{3}$. We say that $K$ is unknotted if it is the boundary of a 2-cell without self-intersections in $S^{3}$. If $K$ is unknotted then $\pi_{1}\left(S^{3}-K\right)$ is free

\footnotetext{
12 Henkelkörper vom Geschlechte $h$ [22, p. 219].
} 
cyclic, and the problem arises naturally: if $\pi_{1}\left(S^{3}-K\right)$ is free cyclic, is $K$ unknotted?

Dehn [4, p. 158, Satz 2], used his lemma to prove that this problem has an affirmative answer, and this is the reason he introduced it.

Another application of Dehn's lemma is made in proving H. Kneser's "Hilfssatz"13 [11, p. 248]. For another application of Dehn's lemma see $[19, \S 5$, pp. 91-92].

However, in my opinion, the greatest importance of Dehn's lemma lies in the fact that it may possibly be used as a tool in proving Poincaré conjecture. Of course this is a personal opinion, and it need not be accepted up to the moment when there will be a proof of Poincaré conjecture based on Dehn's lemma. I would only like to observe that Dehn's lemma is not going to be enough to prove Poincaré conjecture, and that some other things will have to be used too.

12. Applications of the sphere theorem. As we mentioned in No. 3, Eilenberg's paper [5] initiated the asphericity problem of knots, namely: if $K$ is a knot in $S^{3}$, then $S^{3}-K$ is aspherical. This follows easily from the sphere theorem. Moreover the sphere theorem implies Whitehead's conjecture (3.3), see [18, no. 26, pp. 18-19]. Another application of the sphere theorem is made in proving the following statement which was known as Hopf's conjecture. The fundamental group of any open connected subset of the 3-sphere has no element of finite order. In $[18, \$ 7$, pp. $20-23]$, we prove a more general theorem, using the sphere theorem.

In 1948 G. Higman [7, p. 117, No. 1], observed that if $K_{1}, \cdots, K_{m}$ $(m \geqq 2)$ are disjoint knots in $S^{3}$, and if

(12.1) there is a 2-sphere $S$ in $S^{3}$ such that $K_{1}, \cdots, K_{r}$ belong to one component of $S^{3}-S$, and $K_{r+1}, \cdots, K_{m}$ belong to the other component $(1 \leqq r<m)$

then

$$
\begin{aligned}
& \pi_{2}\left(S^{3}-K\right) \neq 0, \quad \text { where } K=K_{1} \cup \cdots \cup K_{m}, \\
& \pi_{1}\left(S^{3}-K\right) \approx A * B,{ }^{4} \text { where } A \text { and } B \neq 1 .
\end{aligned}
$$

Then he proved that (12.3) implies (12.2). Actually his intention was to prove (12.1), which is a much stronger statement. This is proved in $[18$, No. 27, p. 19] and the proof is based on the sphere theorem.

We would like to emphasize, that Higman's remark and problem

13 Several times we have mentioned Kneser's lemma, but we are not going to state it, because it is a rather complicated statement, and we are not going to give any application of it in the present paper. However, we had to mention it, at least for its historical significance. 
are special cases of Kneser's remark and problem (3.2), see also No. 17 .

Other applications of the sphere theorem will be given in $\mathrm{V}$.

\section{RESUltS ON THE CLASSIFICATION PROBLEM}

13. This paper began with the classification problem, it then passed to the three theorems, namely, the sphere theorem, Dehn's lemma, and the loop theorem. Let us now see how far we have gone toward the solution of the classification problem for closed 3-manifolds, using the sphere theorem. Throughout this section we will consider orientable closed 3-manifolds only.

14. Milnor's results. In October $1957 \mathrm{~J}$. W. Milnor [12] obtained some results modulo Poincaré conjecture. To be able to state these results we need some definitions.

Let $M^{\prime}, M^{\prime \prime}$ be two oriented closed 3-manifolds, and let $E^{\prime}, E^{\prime \prime}$ be two open 3-cells in $M^{\prime}, M^{\prime \prime}$ respectively. Matching the boundaries of $M^{\prime}-E^{\prime}$ and $M^{\prime \prime}-E^{\prime \prime}$, in the proper way, we obtain a new oriented closed 3-manifold $M$, called the composition ${ }^{14}$ of $M^{\prime}$ and $M^{\prime \prime}$, and denoted by

$$
M=M^{\prime} \# M^{\prime \prime} .
$$

Homotopy sphere means a simply connected closed 3-manifold; if the Poincaré conjecture is true this is a 3-sphere. Two oriented closed 3-manifolds $M_{1}$ and $M_{2}$ are called congruent if there exist oriented homotopy spheres ${ }^{15} N_{1}$ and $N_{2}$, such that $M_{1} \# N_{1}$ and $M_{2} \# N_{2}$ are isomorphic (i.e. there is an orientation preserving homeomorphism). An oriented closed 3-manifold $M$, which is not a homotopy sphere, is called decomposable if $M$ is congruent to $M^{\prime} \# M^{\prime \prime}$, where neither $M^{\prime}$ nor $M^{\prime \prime}$ is a homotopy sphere. $M$ is called indecomposable if it is not decomposable. Milnor's results are the following.

(14.1) Every oriented closed 3-manifold, which is not a homotopy sphere, is isomorphic to a composition of indecomposable 3-manifolds, which are unique up to order and congruence.

(14.2) Every indecomposable 3-manifold is either congruent to an oriented $S^{1} \times S^{2}$, or is aspherical, or has a nontrivial finite fundamental group.

Of course the 3-manifolds congruent to an oriented $S^{1} \times S^{2}$ are completely defined up to homeomorphism, if Poincaré conjecture

${ }^{14}$ Summenbildung [22, p. 218, Problem 3].

${ }^{15}$ Here is a place where Poincaré conjecture comes into play. Actually we should have called $M_{1}$ and $M_{2}$ congruent (modulo the class of homotopy spheres), but we dropped the parenthesis for abbreviation. 
holds. However the problem is: what are the orientable closed 3-manifolds, which are either aspherical, or their fundamental group is nontrivial and finite?

Milnor obtains his results using the sphere theorem.

15. 3-spheres with handles. We are now going to explain some results due to this author $[18, \S 8$, pp. 23-24].

Let us consider two solid tori of the same genus ${ }^{16} h>0$. Identifying their boundaries in various ways we obtain a denumerable collection of orientable closed 3-manifolds, see No. 10. In this collection we have two extreme identifications. Namely, the identification can be such that the result is a 3-sphere, or such that the result is the duplication ${ }^{\mathbf{1 7}}$ $M_{h}$ of the solid torus of genus $h$. It is easily seen that, $M_{h}$ is the composition of $h$ copies of $S^{1} \times S^{2}$. Moreover $M_{h}$ can also be obtained in the following way: we consider a 3 -sphere $S^{3}$, and $2 h$ disjoint open 3 -cells in it. We then delete the interior of these 3-cells, and identify the boundary of the $i$ th 3 -cell with that of the $(i+h)$-th 3 -cell, $i=1, \cdots, h$, in such a way that the resulting closed 3 -manifold is orientable. This construction justifies the name 3-sphere with $h$ handles given to $M_{h}$.

We observe that $\pi_{1}\left(M_{h}\right)$ is a free group on $h(>0)$ free generators. The question arises naturally: if the fundamental group of an orientable closed 3-manifold is a free group on $h(>0)$ free generators is then this a 3 -sphere with $h$ handles? In my paper [18, \$8, pp. 23-24] an affirmative answer is given to this problem, but only modulo Poincaré conjecture; the proof is based on the sphere theorem, see No. 19.

Let us now consider the following diagram

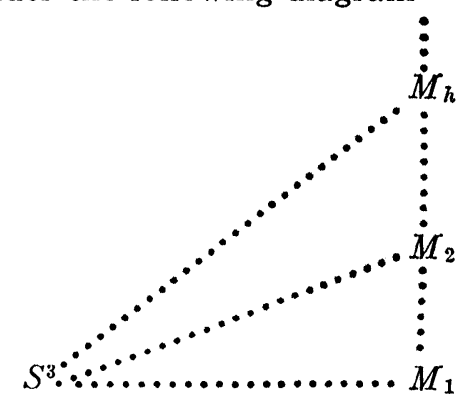

where the dots connecting $S^{3}$ and $M_{h}, h=1,2, \cdots$, represent the orientable closed 3-manifolds we obtain matching the boundaries of two solid tori of genus $h$, in a way different from the two extreme ways that give us $S^{3}$ and $M_{h}$. The 3-manifolds $S^{3}, M_{1}, M_{2}, \cdots$,

16 The case $h=0$ will give us the 3 -sphere.

17 Verdoppelung [22, p. 129]. 
$M_{h}, \ldots$ are characterized up to homeomorphism by their fundamental groups, modulo Poincaré conjecture. The 3-manifolds represented by the horizontal dots are the lens spaces. They have been classified since 1936 by K. Reidemeister [20] modulo the Hauptvemutung, proved in 1952 by E. E. Moise [13, p. 96, Theorem 4]. Another classification of the lens spaces was given in 1949 by R. H. Fox $[6$, p. 455, 11. 22-29], using a certain combinatorial invariant of his own, which was proved by E. J. Brody [3], without use of the Hauptvermutung, to be a topological invariant.

Thus, the 3-manifolds lying on the boundary of the diagram (15.1) are classified, and characterized up to homeomorphism (by algebraic tools), modulo Poincare conjecture. Hence the problem is: what are the 3-manifolds lying in the interior of the diagram (15.1), and how can they be characterized up to homeomorphism (by algebraic tools)?

We would like to point out that, if $M$ is an orientable closed 3manifold, such that $\pi_{1}(M)$ is a free group on $h=h^{\prime}+h^{\prime \prime}$ free generators, then there is a 2 -sphere $S$ in $M$, such that $M-S$ consists of two components $M^{\prime}$ and $M^{\prime \prime}$, where $\pi_{1}\left(M^{\prime}\right)$ and $\pi_{1}\left(M^{\prime \prime}\right)$ are free groups on $h^{\prime}$ and $h^{\prime \prime}$ free generators respectively. ${ }^{18}$ This means that in this case we can solve Kneser's problem (3.2), see also No. 17.

16. The genus of a 3-manifold. An orientable closed 3-manifold may appear on several of the dotted lines ${ }^{19} S^{3} M_{h}$ of the diagram (15.1). The smallest of those numbers $h$ is called the genus $g$ of the 3-manifold, i.e. a 3-manifold is of genus $g$ if it can be obtained by matching the boundaries of two solid tori of genus $g$, but cannot be obtained by matching the boundaries of two solid tori of genus $<g$, $[23$, p. 90, No. 5$]$.

The problem of the genus (i.e. to determine the genus) of an orientable closed 3-manifold seems to be of importance for the classification problem. This is an unsolved problem, and we can solve this problem only, modulo Poincare conjecture, in the special case where the fundamental group of the 3-manifold is a free group on $h \geqq 0$ free generators. Actually by No. 15 , the 3 -manifold is a 3 -sphere with $h$ handles if $h>0$, and so its genus is $h$. However if $h=0$, the 3 -manifold is simply connected, and here is a place where the Poincaré conjecture comes into play. Indeed, Poincaré conjecture asserts nothing else but that any simply connected closed 3-manifold has genus zero.

Another related problem is the following uniqueness problem.

(16.1) Does any orientable closed 3-manifold appear more than once

${ }^{18}$ This can easily be proved, using the method explained in $[18, \S 8, \mathrm{pp} .23-24]$.

19 The end points $S^{3}$ and $M_{h}$ included. 
on a dotted line ${ }^{19} S^{3} M_{h}$ of the diagram (15.1)?

Let us look at this problem more closely. Let $M$ be an orientable closed 3-manifold, and let $F^{\prime}$ and $F^{\prime \prime}$ be two orientable closed surfaces of the same genus $h \geqq 0$ in $M$, such that $M-F^{\prime}$ and $M-F^{\prime \prime}$ consist of two components, the closure of each one of which is a solid torus of genus $h$. Then the uniqueness problem is the following.

(16.2) Does there exist a homeomorphism of $M$ onto itself carrying $F^{\prime}$ onto $F^{\prime \prime}$ ?

We can also pose a stronger problem. $F^{\prime \prime}$ ?

(16.3) Does there exist an isotopy of $M$ into itself carrying $F^{\prime}$ onto

Of course if $h=0$ the answer to both (16.2) and (16.3) is affirmative, see D. E. Sanderson [21]. But what happens if $h>0$ ? It seems that, not even the following special case $M=S^{3}$ is known.

(16.4) Let $F^{\prime}$ and $F^{\prime \prime}$ be two closed surfaces of the same positive genus in $S^{3}$, such that the closure of each one of the components of $S^{3}-F^{\prime}$ and $S^{3}-F^{\prime \prime}$ is a solid torus. Does there exist an isotopy of $S^{3}$ into itself carrying $F^{\prime}$ onto $F^{\prime \prime}$, or at least does there exist a homeomorphism of $S^{3}$ onto itself carrying $F^{\prime}$ onto $F^{\prime \prime}$ ?

17. Kneser's method. We would like to mention here the method developed in the 1928 paper of Kneser [11, No. 4, pp. 252-256], because it seems to me to be of importance. However we have to observe that it contains gaps at some crucial places so that the reader of it has to be especially careful.

Let us mention once again the problem (3.2), which we met in Nos. 12 and 15, in conjunction with the Higman's problem and the 3spheres with handles respectively. We call this problem Kneser's conjecture, and it would be desirable to have a proof of it.

KNESER's CONJECTURE. Let $M$ be an orientable 3-manifold, such that ${ }^{4}$

$$
\pi_{1}(M) \approx A * B .
$$

Then there exist a 2-sphere without singularities $S$ in $M$, such that $M-S$ consists of two components $M^{\prime}$ and $M^{\prime \prime}$, where $\pi_{1}\left(M^{\prime}\right) \approx A$ and $\pi_{1}\left(M^{\prime \prime}\right) \approx B$.

See also No. 20 .

VI. REMARKS

18. Poincaré conjecture and classification. We would like to emphasize the importance of Poincare conjecture for the classification problem of orientable closed 3-manifolds. This is obvious from Nos. 14-16, where the central rôle of this conjecture can be recognized. 
We now observe that using the sphere theorem we obtained some results on the classification problem of orientable closed 3-manifolds. Possibly, using instead of 2-spheres orientable closed surfaces of genus $>0$ in the 3 -manifolds, we might obtain additional information on the classification problem. See [18, §9, p. 24, Problem 3].

19. Geometric problems and algebraic techniques. It seems that the following is true.

(19.1) To solve a geometric problem we need to perform certain geometric operations. However these operations will be possible only under certain conditions. To prove the existence of these conditions, we often need to use algebraic techniques and results.

This needs some explanation. For this reason let us look closely at some of the places in [18], and at the history of some of the problems contained in [18].

To prove his lemma [4, §3, pp. 147-153], Dehn introduced in 1910 a certain geometric operation, which he called Umschaltung (cut) $\left[18\right.$, No. 2, p. 3]. Using the cuts carelessly, ${ }^{20}$ he thought that he had proved his lemma. However what he actually did was, that from the 2-cell with singularities, whose genus is zero, he obtained a surface with fewer singularities, but whose genus might be zero or one. So, though he reduced the singularities, he might have increased the genus. This is a typical mistake in this kind of problem. Namely, to reduce some kind of difficulty, and at the same time to insert a worse difficulty.

I. Johansson, studying diagrams, i.e. the inverse images of Dehn discs, ${ }^{9}$ during the thirties, arrived at the conclusion, that Dehn's lemma might be proved possibly, by conveniently selected cuts $[8$, p. 314 , 11. 18-20]. His conclusion is justified by this author's proof, for the case of an orientable 3-manifold.

Actually, looking closer at the proof of Dehn's lemma in [18], we observe that we actually construct the desired disc $[18$, p. $2,11$. 34-38], and that the construction is carried out by means of successive cuts. Let us now analyze our proof, and point out the delicate points.

Using covering spaces of covering spaces (the tower) No. 8, we arrive at a crucial value $n$ (the height) where the process actually stops. Obviously, here something has to be brought into play, and this is a theorem due to H. Seifert [22, p. 223, Satz IV], that the first betti number, of a compact 3-manifold with boundary, is at least equal to the sum of the genera of the boundary surfaces. This is a key theorem for

${ }^{20}$ Actually that time was the early days of Topology, and the difficulties of its problems had not been generally realized. 
3-manifolds, relating an algebraic notion (the betti number) with a geometric one (the genus). Seifert's theorem implies the following, see diagram (8.4).

(19.2) All boundary surfaces of $V_{n}$ are 2-spheres.

This is really decisive, as we shall see below. We now have to distinguish the following two cases $d\left(D_{n}\right)>$ or $=0$, see No. 8 .

In case $d\left(D_{n}\right)>0$, see $[18$, No. 16, pp. 14-15], the process performed at the $n$th level is based on (19.2), and its projection by the map $p_{1} \cdots p_{n}$ is essentially a certain finite number of cuts of the Dehn disc, which do not increase the genus of it, but decrease the complexity. But, how could we find the cuts needed, without the use of covering spaces and (19.2)?

In case $d\left(D_{n}\right)=0$ the above process cannot be applied any more. So some new process has to be found. The intuition suggests that there should exist a double curve on the Dehn disc $D$, which is a simple $e^{3}$ curve. This actually holds, and the proof of it is rather algebraic, see [18, Nos. 11-12, pp. 11-13]. We emphasize that this is the most delicate part of the proof. After the proof of the existence of such a curve, things are easy. In fact we perform a cut of the Dehn disc $D$, along that curve, and we obtain a new Dehn disc with less complexity. The cut along a "simple" double curve is not dangerous, i.e. it does not increase the genus of the surface. This is the only case where we can apply Dehn's process, without any danger.

Looking back we see that the main difficulty of the problem was to prove the existence of nondangerous cuts, and to prove this existence we used some algebraic results and techniques, at some crucial places. The above justifies (19.1).

We can have analogous remarks and conclusions, analyzing the proof of the sphere theorem. However, the geometric operations needed here are the cuts and some carefully performed deformations. We have to keep in mind that we need the additional condition, that the 2-sphere is not homotopic to zero in the 3-manifold.

We now turn to an analysis of the proof of Theorem (32.1), [18, p. 23]. This theorem asserts that, if Poincare conjecture is true, then any orientable closed 3-manifold, whose fundamental group is a free group on $h(\geqq 0)$ free generators, is a 3-sphere with $h$ handles. The proof is by induction on $h$, and makes use of the sphere theorem. However, to be able to apply the sphere theorem we need to prove that $\pi_{2} \neq 0$. This is done by using a theorem due to E. Specker [24, p. 325, Satz VI]. The proof of this last theorem is based on the first part of Specker's paper, which makes use of the theories of B. Eckmann, H. Freudenthal, H. Hopf, W. Hurewicz, and others. It is difficult to 
see how we could go through, without the knowledge of those algebraic topological theories. A final remark is the following: in the proof of the above theorem, the induction starts with $h=1$, and the theorem for $h=0$ is Poincaré conjecture. Once again we see the importance of this conjecture.

We hope that the above explanations clarify and justify (19.1).

20. Kneser's conjecture. At several places in this paper we met Kneser's conjecture, see Nos. 3, 12, 15, and especially No. 17. Let us now look closer at the proof of Higman's problem, which is a special case of Kneser's conjecture as was observed in No. 12. We would like to prove that (12.3) implies (12.1), and we proved this by proving first that (12.3) implies (12.2), and secondly that (12.2) implies (12.1). The first was proved by G. Higman [7, p. 122, Theorem 2], using algebraic techniques, and the second was proved by this author using the sphere theorem [18, No. 27, p. 19]. This suggests that the gap between (17.1) and the conclusion of Kneser's conjecture is so great, that it has to be factored, and we first have to prove that (17.1) implies $\pi_{2} \neq 0$, and then that $\pi_{2} \neq 0$ implies the desired conclusions. It seems that the first step has to be proved by algebraic topological techniques, and the second one by using the sphere theorem and something more, because the sphere theorem is not enough to provide us with the conclusions of Kneser's conjecture. Thinking now that the algebraic topological techniques were rather undeveloped in 1928 , we easily conclude that it was rather hopeless, to expect to have a satisfactory proof of this strong statement at that time.

21. Nonclosed 3-manifolds. It is known that any simply connected open ${ }^{21}$ surface is an open 2-cell [22, p. 320, Remark 26]. However such a theorem does not hold for 3-dimensions. In $1935 \mathrm{~J}$. H. C. Whitehead $[25 ; 26]$ constructed an open 3 -manifold which has the homotopy type of an open 3-cell, ${ }^{22}$ but which is not an open 3-cell, $[15, \mathrm{p} .19$, Theorem 3 ]. This example is enough to indicate how complicated things are for 3-manifolds if we drop compactness. The example also indicates, that we do not have the analogue of Poincaré conjecture for open 3manifolds.

As far as the 3-manifolds with boundary, compact or not, are concerned, we restrict ourselves to the consideration of the following special case. Let $K$ be a knot in $S^{3}$, and let $T$ be a small tube around

${ }^{21}$ Open means noncompact and without boundary.

22 It has also the additional property, that any 2-sphere (without singularities) in it bounds a 3-cell (without singularities) in it. 
$K$. Then the closure of $S^{3}-T$ is a compact 3-manifold $M_{K}$ whose boundary is the same as that of $T$. The classification of the $M_{K}$ 's is equivalent to the classification of the knots in $S^{3}$, and this is supposed to be a very difficult problem. We now observe that $M_{K}$ is a compact 3-manifold with boundary, of a very special kind. Namely $M_{K}$ can be imbedded in $S^{3}$, and its boundary is a closed surface of genus one.

From the above, one gets an idea of how difficult the classification problems are for nonclosed 3-manifolds.

22. We would like to point out that the purpose of this paper is not to give a complete account of the knowledge of the classification problem of 3-manifolds. Many nice and important things have not been even mentioned in this paper. The purpose of this paper is only to explain the point of view in which this author believes.

\section{BiBLIOGRAPHY}

1. R. H. Bing, Locally tame sets are tame, Ann. of Math. vol. 59 (1954) pp. 145-158.

2. - An alternative proof that 3-manifolds can be triangulated, Ann. of Math. (to appear).

3. E. J. Brody, On the Fox invariant, Dissertation, 1954, Princeton University.

4. M. Dehn, Über die Topologie des dreidimensionalen Raumes, Math. Ann. vol. 69 (1910) pp. 137-168.

5. S. Eilenberg, Sur les courbes sans noeuds, Fund. Math. vol. 28 (1936) pp. 233242.

6. R. H. Fox, Recent development of knot theory at Princeton, Proceedings of the International Congress of Mathematicians 1950, vol. II, pp. 453-457.

7. G. Higman, A theorem on linkages, Quart. J. Math. Oxford Ser. vol. 19 (1948) pp. $117-122$.

8. I. Johansson, Über singuläre Elementarflächen und das Dehnsche Lemma, Math. Ann. vol. 110 (1935) pp. 312-320.

9. - - Über singuläre Elementarflächen und das Dehnsche Lemma II, Math. Ann. vol. 115 (1938) pp. 658-669.

10. B. v. Kerékjárto, Vorlesungen ïber Topologie, Berlin, Springer, 1923.

11. H. Kneser, Geschlossene Flächen in dreidimensionalen Mannigfaltigkeiten, Jber. Deutsch. Math. Verein. vol. 38 (1929) pp. 248-260.

12. J. W. Milnor, A unique decomposition theorem for 3-manifolds (in preparation).

13. E. E. Moise, Affine structures in 3-manifolds V, Ann. of Math. vol. 56 (1952) pp. 96-114.

14. —- Affine structures in 3-manifolds VIII, Ann. of Math. vol. 59 (1954) pp. 159-170.

15. M. H. A. Newman and J. H. C. Whitehead, On the group of a certain linkage, Quart. J. Math. Oxford Ser. vol. 8 (1937) pp. 14-21.

16. C. D. Papakyriakopoulos, On solid tori, Proc. London Math. Soc. (3) vol. 7 (1957) pp. 281-299.

17. - On Dehn's lemma and the asphericity of knots, Proc. Nat. Acad. Sci. U.S.A. vol. 43 (1957) pp. 169-172.

18. - On Dehn's lemma and the asphericity of knots, Ann. of Math. vol. 66 (1957) pp. 1-26. 
19. - On the ends of the fundamental groups of 3-manifolds with boundary, Comment. Math. Helv. vol. 32 (1957) pp. 85-92.

20. K. Reidemeister, Homotopieringe und Linsenräume, Abh. Math. Sem. Hansischen Univ. vol. 11 (1935) pp. 102-109.

21. D. E. Sanderson, Istopic deformations in 3-manifolds, Thesis, 1953, University of Wisconsin.

22. H. Seifert and W. Threlfall, Lehrbuch der Topologie, Leipzig, Teubner, 1934.

23. J. Singer, Three-dimensional manifolds and their Heegaard diagrams, Trans. Amer. Math. Soc. vol. 35 (1933) pp. 88-111.

24. E. Specker, Die erste Cohomologiegruppe von Überlagerungen und Homotopieeigenschaften dreidimensionaler Mannigfaltigkeiten, Comment. Math. Helv. vol. 23 (1949) pp. 303-332.

25. J. H. C. Whitehead, A certain region in euclidean 3-space, Proc. Nat. Acad. Sci. U.S.A. vol. 21 (1935) pp. 364-366.

26. - A certain open manifold whose group is unity, Quart. J. Math. Oxford Ser. vol. 6 (1935) pp. 268-279.

27. - On doubled knots, J. London Math. Soc. vol. 12 (1937) pp. 63-71.

28. - On the asphericity of regions in a 3-sphere, Fund. Math. vol. 32 (1939) pp. $149-166$.

29. - 2-spheres in 3-manifolds, Bull. Amer. Math. Soc. vol. 64 (1958) pp. 161-166.

30. J. H. C. Whitehead and Arnold S. Shapiro, $A$ proof and extension of Dehn's lemma, Bull. Amer. Math. Soc. vol. 64 (1958) pp. 174-178.

InstituTe for AdVANCEd STUdy 